\title{
Computational results for the known biplanes of order 9
}

\author{
J. D. Key* \\ Department of Mathematical Sciences \\ Clemson University \\ Clemson SC 29634 \\ V. D. Tonchev \\ Department of Mathematical Sciences \\ Michigan Technological University \\ Houghton MI 49931-1295
}

\begin{abstract}
Using Magma we examine the ternary codes associated with the five known biplanes of order 9. The computations showed that each biplane is the only one to be found among the weight-11 vectors of its ternary code, and that none of the biplanes can be extended to a 3 - $(57,12,2)$ design. We examined also all the residual designs of the biplanes, and the designs associated with $\{12 ; 3\}$-arcs.
\end{abstract}

\section{Introduction}

There are five known biplanes of order 9, i.e. 2-(56,11,2) designs. Following the notation commenced by Denniston [8] we denote these by $B 1$ to $B 5$ where

1. B1 was found by Hall, Lane and Wales [10];

2. B2 was found by Mezzaroba and Salwach [14];

3. B3 was found by Denniston [8];

*Support of NSF grant GER-9450080 acknowledged 
4. B4 was found by Denniston [8];

5. B5 was found by Janko and Trung [12].

The order of a biplane of block size 11 is 9 , and thus only the ternary codes of such a biplane will be of interest. We computed the dimensions of the code of the design, its orthogonal (dual) code, the hull (the intersection of the code and its orthogonal), and the orthogonal of the hull, in each case, and found the minimum weight of each code. We also collected the minimum words of the code of each of these designs; in all cases 11 is the minimum weight, and in all but one case the only minimum words were the incidence vectors of the blocks of the design. In the case of $B 3$ there were more weight- 11 vectors, but they were not constant vectors, and even so, the supports did not hold another biplane.

We also collected all (or almost all) of the constant words of weight 12 in the orthogonal code for each of the biplanes that had sufficient of these, since this would allow us to be able to find out whether or not any of these biplanes could be extended (see the argument of why this holds in Section 2). In fact three of the biplanes (B3,B4 and $B 5)$ had insufficient numbers of such vectors, and the other two we were able to check and show that there are not enough with the required intersection properties. Thus we conclude that none of the biplanes can be extended to a $3-(57,12,2)$ design. This confirmed various earlier claims for the first four biplanes that we have been unable to find documented proof of, as well as the findings of Bagchi [2], who claimed to have a proof that designs with these parameters cannot exist. This claim was subsequently invalidated by Brouwer [4] (see also Brouwer and Wilbrink [5]) due to a computational error (Lemma 4.1 in [2], which is crucial to the proof, asserts that a certain $16 \times 16$ matrix is nonsingular which leads to a unique solution of a system of equations, yielding a contradiction that establishes the lemma; the matrix however has rank 15, as was shown by Brouwer and verified again by our computations with Magma). Note that the interest in the extendability of biplanes with the given parameters stems partially from Cameron's theorem [6] (see [11, Theorem 4.2] for a statement and proof) which singles out this case as being a possible candidate of a symmetric design extending to a 3-design.

All the computations concerning the ternary codes and the collection of the vectors were made using Magma [3]; the computations to check the supports of the weight-12 vectors in the case of $B 1$ and $B 2$ were made initially at Michigan Technological University, and later checked with Magma. In the table $C$ denotes the ternary code of the biplane, and $H$ its hull; the 


\begin{tabular}{||c||c|r||r|r|r|r||c||c||}
\hline \hline Biplane & $\operatorname{dim}(C)$ & \multicolumn{1}{|c|}{$\mid$ Aut $\mid$} & \multicolumn{3}{|c||}{ Minimum weight } & Wt 11 & Wt 12 \\
\hline & & & $C$ & $C^{\perp}$ & $H$ & $H^{\perp}$ & $C$ & $C^{\perp}$ \\
\hline \hline$B 1$ & 20 & 80640 & 11 & 8 & 18 & 7 & 112 & 2100 \\
\hline$B 2$ & 22 & 288 & 11 & 8 & 12 & 7 & 112 & 516 \\
\hline$B 3$ & 26 & 144 & 11 & 8 & 12 & 7 & 120 & $\leq 91$ \\
\hline$B 4$ & 24 & 64 & 11 & 8 & 12 & 7 & 112 & 148 \\
\hline$B 5$ & 26 & 24 & 11 & 8 & 12 & 7 & 112 & $\leq 22$ \\
\hline \hline
\end{tabular}

Table 1: The five biplanes

last two columns show the number of minimum weight words of $C$ and the number of $\{0,1\}$ weight-12 words of $C^{\perp}$, respectively.

The paper is organised as follows: after a brief description of our terminology and some background results, Sections 3 deals with the nonextendability of the five biplanes; Section 4 gives the results we obtained for the 16 residual designs; Section 5 the results we obtained for $\{12 ; 3\}$ arcs; and the final section is by way of an appendix, giving some weight distributions and complete weight enumerators.

\section{Background and terminology}

The notation used is generally standard and we refer the reader to Assmus and Key [1]. We recall here some of the definitions that we particularly need.

An incidence structure $\mathcal{D}=(\mathcal{P}, \mathcal{B})$ with point set $\mathcal{P}$ and block set $\mathcal{B}$ is a $t-(v, k, \lambda)$ design if every block is incident with precisely $k$ points and any set of $t$ distinct points are together incident with precisely $\lambda$ blocks. It follows (see [1, Chapter 1]) that $\mathcal{D}$ is an $s$-design for any $s<t$; we denote the number of blocks incident with $s$ points by $\lambda_{s}$. The order of a $t$-design, where $t \geq 2$, is $n=\lambda_{1}-\lambda_{2}$. Given a $t$ - $(v, k, \lambda) \operatorname{design} \mathcal{D}=(\mathcal{P}, \mathcal{B})$, let $x \in \mathcal{P}$; the derived structure at $x$ is defined to be the incidence structure $\mathcal{D}_{x}=\left(\mathcal{P}-\{x\}, \mathcal{B}_{x}\right)$, where $\mathcal{B}_{x}=\{B \mid B \in \mathcal{B}, x \in B\}$, with incidence as in $\mathcal{D}$. If $t \geq 2$ then $\mathcal{D}_{x}$ is a $(t-1)-(v-1, k-1, \lambda)$ design. If $\mathcal{D}$ and $\mathcal{E}$ are designs and $\mathcal{D}=\mathcal{E}_{x}$ for some point $x$ in the point-set of $\mathcal{E}$, then $\mathcal{E}$ is called an extension of $\mathcal{D}$. 
A symmetric design has $|\mathcal{P}|=|\mathcal{B}|$, and is usually denoted simply by the parameters $(v, k, \lambda)$. The symmetric designs with $\lambda=1$ are the finite projective planes; symmetric designs with $\lambda=2$ are called biplanes. The order of a biplane with block size $k$ is $k-2$. Unlike the situation for planes, there are only a small finite number of biplanes known at present, and it is completely unknown if the number of biplanes is finite or infinite. For $k=11$ there are five biplanes known, but this list is not known to be complete. The parameters in this case satisfy the necessary divisibility conditions for the biplanes to extend to $3-(57,12,2)$ designs. However, no such designs are known to exist and we have now shown computationally that the five known biplanes cannot be extended.

For a symmetric design $\mathcal{D}$, the dual structure is also a symmetric design, and we denote it by $\mathcal{D}^{t}$. In this case we can also define a residual design for any block $B$ of $\mathcal{D}$ : this is the incidence structure $\mathcal{D}^{B}=\left(\mathcal{P}-B, \mathcal{B}^{B}\right)$ where where $\mathcal{B}^{B}=\{C-B \mid C \in \mathcal{B}-\{B\}\}$. Thus the residual is obtained by deleting one block and all the points on it: for example, affine planes are obtained from projective planes by precisely this method. Thus if $\mathcal{D}$ is a symmetric $2-(v, k, \lambda)$ design, then $\mathcal{D}^{B}$ is a $2-(v-k, k-\lambda, \lambda)$ design. Conversely, in the case of $\lambda=1$ or 2 , a $2-(v-k, k-\lambda, \lambda)$ design can be uniquely embedded in a symmetric design: for $\lambda=1$ the result is classical and can be found in any textbook on geometry ( or see, for example, Hughes and Piper [11]) and for biplanes the result is due to Connor and Hall [9].

For any field $F, F^{\mathcal{P}}$ is the vector space of functions from $\mathcal{P}$ to $F$ with basis given by the characteristic functions of the singleton subsets of $\mathcal{P}$. If $\mathcal{D}=(\mathcal{P}, \mathcal{B})$ is an incidence structure, the code $C_{F}(\mathcal{D})$ of $\mathcal{D}$ over $F$ is the subspace of $F^{\mathcal{P}}$ spanned by the characteristic functions (incidence vectors) of the blocks of $\mathcal{D}$. If $X \subseteq \mathcal{P}$, denote the characteristic function on $X$ by $v^{X}$ : thus

$$
v^{X}(x)=\left\{\begin{array}{ll}
1 & \text { if } x \in X \\
0 & \text { if } x \notin X
\end{array},\right.
$$

where $v^{X}(x)$ denotes the value that the function $v^{X}$ takes at the point $x$. Then

$$
C_{p}(\mathcal{D})=\left\langle v^{B} \mid B \in \mathcal{B}\right\rangle .
$$

The dimension of $C_{p}(\mathcal{D})$ is referred to as the $p$-rank of $\mathcal{D}$. It is well known that the code of a design of order $n$ will only be of any interest or use in characterizations of the design when the prime $p$ divides $n$ : see [1], for example. 
The orthogonal code $C^{\perp}$ (where the orthogonal is taken with respect to the standard inner product in $F^{v}$, i.e. , for $u, w \in F^{v},(u, w)=$ $\left.\sum_{x \in \mathcal{P}} u(x) w(x),\right)$ is defined by

$$
C^{\perp}=\left\{u \mid u \in F^{v} \text { and }(u, w)=0 \text { for all } w \in C\right\} .
$$

The hull of a design $\mathcal{D}$ with code $C$ over the field $F$ is the code

$$
\operatorname{Hull}_{F}(\mathcal{D})=H_{F}(\mathcal{D})=C \cap C^{\perp} .
$$

Notice that the code $\left\langle v^{B}-v^{C} \mid B, C \in \mathcal{B}\right\rangle$ is inside the hull, and equal to it, with codimension 1 in the code of the design, in this case.

Lemma 1 If a 2-(56,11,2) design extends to a $3-(57,12,2)$ design, then the incidence vectors of the blocks of the 3-design that do not contain the new point must be in the orthogonal of the design's ternary code.

Proof: Since any two blocks of a biplane meet in exactly two points, any two blocks of an extension meet in three or zero points. Since our code is ternary, the result follows.

Thus if we can find the $\{0,1\}$ vectors of weight 12 in the orthogonal code $C^{\perp}$, we must be able to find our new blocks amongst these 12 -sets, i.e. we look for 21012 -sets with the correct properties. Since these codes are rather large, we needed to reduce the time taken for the computation in whatever ways possible. The complete weight enumerator of the code $C^{\perp}$ would indicate the number of constant weight- 12 words. This could be obtained from the complete weight enumerator of the hull in the following way: denoting this polynomial by $H(x, y, z)$, the complete weight enumerator for the code $C$ of the design is given by

$$
C(x, y, z)=H(x, y, z)+H(y, z, x)+H(z, x, y),
$$

since $C=\mathrm{Hull}+\langle\boldsymbol{\jmath}\rangle$.

Now we can use the extension of the MacWilliams relations to complete weight enumerators (see, for example, [1, Chapter 2, p. 84]) to get the complete weight eumerator of $C^{\perp}$ :

$$
D(x, y, z)=\frac{1}{|C|} C\left(x+y+z, x+a y+a^{2} z, x+a^{2} y+a z\right),
$$

where $a$ is a primitive third root of unity. In fact Magma now has a function to perform the transform. 


\section{Non-extendability}

\subsection{The biplane $B 1$}

We showed that the biplane $B 1$ cannot be extended, confirming an earlier claim of a proof of this quoted in [2] as being due to Hall and Baumert. We obtained the complete weight-enumerators of $\operatorname{Hull}(C)$, and thus of all the four codes $C, C^{\perp}, \operatorname{Hull}(C)$ and $\operatorname{Hull}(C)^{\perp}$ using the formulae described above. This showed that the number of constant $\{0,1\}$ vectors of weight-12 in $C^{\perp}$ is 2100 .

Theorem 2 The biplane B1 cannot be extended.

Proof: The proof is by computer. The complete weight enumerator of $C^{\perp}$ (where $C$ is the ternary code of the design) told us that there were 2100 $\{0,1\}$ vectors in that code. Using the group to assist in completing orbits, we collected all the 2100 supports of these words and were able to show that we could not collect 210 to complete the number of blocks for the extended design. This was done by finding all the words through one of the points (450) and then forming a graph with these as vertices and with adjacency defined if the supports meet in three points. We could then find the size of a maximal clique, and this turned out to be 11, instead of 45 as would be required.

In fact the words of weight 12 in the orthogonal code fell into three orbits under the automorphism group, i.e. two of length 420 and one of length 1260. Each of these three orbits formed the blocks of a $2-(56,12, \lambda)$ design, with $\lambda=18$ and 54 , respectively. The two $2-(56,12,18)$ designs so obtained are not isomorphis but each has the automorphism group of $B 1$ as its full automorphism group, as does the 2-(56,12,54) design.

We include in the appendix, Section 6.1, the Magma output showing the weight-distributions of the four codes associated with $B 1$. We include also the complete weight enumerator of $\operatorname{Hull}(C)$, leaving it to the reader to obtain the complete weight enumerators for the code and its orthogonal, using the formulae described above and any accessible algebra package, for example Maple:

\subsection{The biplane $B 2$}

The code of the design has dimension 22. With Magma we were able to obtain the weight distribution of each of its four associated codes, and also 
the complete weight-enumerators in each case, as described for $B 1$. There are 516 constant $\{0,1\}$ weight- 12 vectors in the code $C^{\perp}$; we were able to collect 492 of these and apply the same argument as given in the case of $B 1$ to find that the maximal size of a clique through one of the points is 9 , still short of the required 45, even if all the remaining 24 blocks could be used. We thus showed that we could not extract 210 to complete an extension for $B 2$, and thus obtained

Theorem 3 The biplane B2 cannot be extended.

The Magma output for the weight distributions for B2's codes can be found in Section 6.2.

\subsection{The biplane $B 4$}

The code of the design has dimension 24. With Magma we were able to obtain the complete weight enumerator of the hull of $B 4$, of dimension 23 . This took approximately 13 days (1131129.444 seconds). Using the formulae given in Section 2, as before, we obtained the complete weight enumerators of the code, its orthogonal and also the hull's orthogonal. This yielded that there are only $148\{0,1\}$ weight- 12 vectors in the code $C^{\perp}$, and thus not sufficient for the design to extend.

Theorem 4 The biplane B4 cannot be extended.

The Magma output for the weight distributions for B4's codes is given in Section 6.3.

\subsection{The biplanes $B 3$ and $B 5$}

The ternary hulls of these two biplanes have dimension 25; noting that the 23-dimensional hull of $B 4$ required 13 days for magma to obtain the complete weight enumerator, we deduce that the complete weight enumerator for either of these would take 130 days, i.e. rather too long. Expecting that the orthogonal codes would not in fact have sufficient $\{0,1\}$ vectors of weight 12, the following method was attempted: instead of taking the span of all the blocks, a subset was chosen to span a code of lower dimension that did not contain the all-one vector. Thus if $C_{1}$ denotes this code, of dimension $d$, then $C_{2}=C_{1}+\langle\boldsymbol{j}\rangle$ has dimension $d+1$ and its orthogonal code $C_{2}^{\perp}$ will contain the design's orthogonal code $C^{\perp}$. The complete weight enumerator 
of $C_{1}$ could be found in at most 13 days if $d \leq 23$, and this would yield the complete weight enumerator of $C_{2}^{\perp}$ quite quickly, by the method described in Section 2. In fact $d=22$ for $B 3$ yielded $C_{2}^{\perp}$ with only $91\{0,1\}$ vectors of weight 12 , and $d=22$ for $B 5$ gave only 22 of the required vectors in $C_{2}^{\perp}$. We may conclude that in neither case does $C^{\perp}$ have sufficient weight-12 $\{0,1\}$ vectors. Thus we have

Theorem 5 The biplanes B3 and B5 cannot be extended.

A partial search amongst the weight- $12\{0,1\}$ vectors in the two orthogonal codes yielded that $B 3$ had at least 42 , and $B 5$ at least 10 , such vectors.

\section{The residual designs}

The five biplanes yield 16 non-isomorphic residual 2-(45, 9, 2) designs (affine designs); the 16 ternary codes are also non-isomorphic. The number of different affine designs corresponds to the number of orbits on blocks:

1. B1 has one;

2. B2 has three;

3. B3 has three;

4. B4 has four;

5. B5 has five.

Table 2 shows, for each of the 16 affine designs, the size of the automorphism group, the dimension of the ternary code, the minimum weight of the code, the number of weight-9 vectors in the code, and the minimum weight of the orthogonal code, respectively.

The affine design for $B 1$ (there is only one since $B 1$ has a transitive automorphism group) has the smallest code, and thus the biggest $H^{\perp}=$ $C+C^{\perp}$. This latter code (of dimension 36) had many (i.e. 140135) constant $\{0,1\}$ weight-9 vectors, as the complete weight enumerator showed, and it is possible that other designs with these parameters might be found amongst these vectors. 


\begin{tabular}{|c||c|c|c|c|c||}
\hline \hline Design & $|A u t|$ & $\operatorname{dim}(C)$ & Min Wt. $C$ & Wt-9 Wds in $C$ & Min Wt. $C^{\perp}$ \\
\hline \hline$B 1$ & 1440 & 19 & 9 & 110 & 8 \\
\hline$B 2: 1$ & 144 & 21 & 6 & 110 & 8 \\
$B 2: 2$ & 16 & 21 & 8 & 126 & 8 \\
$B 2: 3$ & 8 & 21 & 8 & 126 & 8 \\
\hline$B 3: 1$ & 72 & 25 & 6 & 406 & 10 \\
$B 3: 2$ & 8 & 25 & 8 & 494 & 10 \\
$B 3: 3$ & 4 & 25 & 6 & 438 & 8 \\
\hline$B 4: 1$ & 16 & 23 & 6 & 158 & 8 \\
$B 4: 2$ & 16 & 23 & 7 & 142 & 8 \\
$B 4: 3$ & 4 & 23 & 7 & 186 & 8 \\
$B 4: 4$ & 2 & 23 & 7 & 198 & 8 \\
\hline$B 5: 1$ & 3 & 25 & 8 & 278 & 8 \\
$B 5: 2$ & 4 & 25 & 7 & 370 & 8 \\
$B 5: 3$ & 1 & 25 & 7 & 318 & 8 \\
$B 5: 4$ & 2 & 25 & 6 & 398 & 8 \\
$B 5: 5$ & 4 & 25 & 6 & 370 & 8 \\
\hline
\end{tabular}

Table 2: The 16 residual designs 


\section{Some maximal $\{12 ; 3\}$-arcs}

The support of a weight-12 vector in the orthogonal code of any of the five biplanes will have the property that any block will meet it in zero or three points, and thus will be a (maximal) $\{12 ; 3\}$-arc for the biplane (in the terminology of Denniston[8] and Morgan[13]). This is clear from a quick counting argument. Conversely, any $\{12 ; 3\}$-arc in the biplane is the support of a $\{0,1\}$ weight- 12 vector in the orthogonal code. Furthermore, taking the intersections with such an arc of all the blocks that meet the arc in three points will give a $2-(12,3,2)$ design. Blocks of these designs will meet in zero, one or two points. We have candidates here for class-3 association schemes:

Definition $1 A n$ association scheme of class $d$ is a set $X$ together with a partition of its subsets of size 2 into $d$ non-empty classes $\Gamma_{1}, \ldots, \Gamma_{d}$ satisfying the two conditions:

(i) for $x \in X$ and $i \in\{1, \ldots, d\}$, the number $n_{i}(x)$ of points $y \in X$ with $\{x, y\} \in \Gamma_{i}$ depends on $i$ and not on $x$; thus write this number as $n_{i}$;

(ii) for $x, y \in X$ and $\{x, y\} \in \Gamma_{k}$, the number $p_{i, j}^{k}(x, y)$ of points $z \in X$ with $\{x, z\} \in \Gamma_{i}$ and $\{y, z\} \in \Gamma_{j}$ depends only on $i, j, k$ and not on $x$ and $y$; thus write this number $p_{i, j}^{k}$.

(See, for example, Cameron and van Lint [7, Chapter 17] for more on association schemes.)

We computed these designs, their automorphism groups and ternary codes for all the $\{12 ; 3\}$-arcs that we collected. The set of 44 blocks of any of these $2-(12,3,2)$ designs is a candidate for an association scheme of class 3 , by taking $\Gamma_{i}$ to be the set of all pairs of blocks that meet in $i$ points, where $i=0,1,2$. Condition $(i)$ of the definition is satisfied (counting gives $n_{1}=16, n_{2}=24, n_{3}=3$ ), but not $(i i)$ for any of the $\{12 ; 3\}$-arcs and thus none of these give class- 3 association schemes. We found designs in $B 1$ isomorphic to some in $B 2$ and $B 5$.

\section{Magma output of weight distributions}

\subsection{The biplane $B 1$}

//weight distribution of code (dim=20) 
$[\langle 0,1\rangle,\langle 11,112\rangle,\langle 18,4200\rangle,\langle 20,16744\rangle,\langle 21,8064\rangle,\langle 23$, $312480\rangle,\langle 24,528150\rangle,\langle 26,5962992\rangle,\langle 27,6309520\rangle,\langle 29$, $53245360\rangle,\langle 30,48544272\rangle,\langle 32,247932090\rangle,\langle 33,179524800\rangle,\langle 35$, $618365808\rangle,\langle 36,361395468\rangle,\langle 38,776577200\rangle,\langle 39,357966000\rangle,\langle 41$, $476449344\rangle,\langle 42,170451360\rangle,\langle 44,130974480\rangle,\langle 45,34736016\rangle,\langle 47$, $14241920\rangle,\langle 48,2769480\rangle,\langle 50,425376\rangle,\langle 51,18816\rangle,\langle 53,18480\rangle$, $\langle 54,5320\rangle,\langle 56,548\rangle]$

//weight distribution of code's orthogonal (dim=36)

$[\langle 0,1\rangle,\langle 8,3150\rangle,\langle 10,47376\rangle,\langle 11,53760\rangle,\langle 12,1282680\rangle$, $\langle 13,4929120\rangle,\langle 14,30567600\rangle,\langle 15,142994188\rangle,\langle 16,802027170\rangle$, $\langle 17,3619533900\rangle,\langle 18,15950552520\rangle,\langle 19,63613873098\rangle,\langle 20$, $236151876828\rangle,\langle 21,809344687835\rangle,\langle 22,2578073354640\rangle,\langle 23$, $7619254446065\rangle,\langle 24,20958048548010\rangle,\langle 25,53654145146873\rangle,\langle 26$, $127903637805552\rangle,\langle 27,284263003036745\rangle,\langle 28,588927707457120\rangle$, $\langle 29,1136900859613680\rangle,\langle 30,2046755888776973\rangle,\langle 31$, $3433801054118400\rangle,\langle 32,5363668444107330\rangle,<33$, $7802853216762459\rangle,\langle 34,10558371000234960\rangle,<35$, $13269335870959344\rangle,<36,15483295119369274\rangle,<37$, $16741304199879840\rangle,\langle 38,16736216289069480\rangle,<39$, $15451134136921028\rangle,\langle 40,13135418302138020\rangle,<41$, $10248888825984816\rangle,\langle 42,7321769696007079\rangle,\langle 43$, $4768416874458720\rangle,\langle 44,2816856218473920\rangle,<45$,

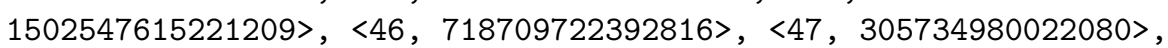
$\langle 48,114668537572426\rangle,\langle 49,37450290764160\rangle,<50$,

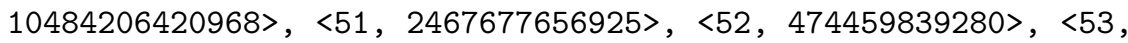
$71455668480\rangle,\langle 54,7633541597\rangle,\langle 55,563774400\rangle,\langle 56,20286852\rangle$ ]

//weight distribution of hull (dim=19)

$[\langle 0,1\rangle,\langle 18,4200\rangle,\langle 21,8064\rangle,\langle 24,528150\rangle,\langle 27,6309520\rangle$, $\langle 30,48544272\rangle,\langle 33,179524800\rangle,\langle 36,361395468\rangle,\langle 39$, $357966000\rangle,\langle 42,170451360\rangle,\langle 45,34736016\rangle,\langle 48,2769480\rangle,\langle 51$, $18816\rangle,\langle 54,5320\rangle]$

//weight distribution of hull`perp (dim=37)

$[\langle 0,1\rangle,\langle 7,480\rangle,\langle 8,3150\rangle,\langle 10,50736\rangle,\langle 11,162960\rangle,\langle 12$, $2492280\rangle,\langle 13,12111120\rangle,\langle 14,80771760\rangle,\langle 15,459754848\rangle,\langle 16$, $2747147239\rangle,\langle 17,11075248800\rangle,\langle 18,48280565777\rangle,\langle 19$, $191569234080\rangle,\langle 20,708759553807\rangle,\langle 21,2430444925152\rangle,<22$, $7731740021826\rangle,\langle 23,22858564806000\rangle,\langle 24,62871989217034\rangle,<25$, 
160923590408784>, <26, 383748523032444>, <27, 852895690654000>, $<28,1766457057420194\rangle,<29,3411064960638720\rangle,<30$, $6140874317404176>,<31,10299227084830150\rangle,<32$, 16092585079793460>, <33, 23410926231809760>, <34, $31668806739230200\rangle,<35,39812168262647808\rangle,<36$, 46454652317997756>, <37, 50213572558513631>, <38, $50213599598116920\rangle,<39,46358049168552240\rangle,<40$, 39398345535719163>, <41, 30749921722760688>, <42, $21967591355842320\rangle,<43,14302293917808695\rangle,<44$, $8451354693720960\rangle,<45,4508072547170640\rangle,<46$,

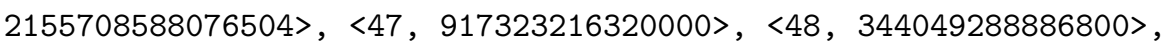
$\langle 49,112324451129270\rangle,\langle 50,31451061932136\rangle,<51$, $7401263772672\rangle,\langle 52,1423852057289\rangle,<53,214746794640\rangle,<54$, $23877991480\rangle,\langle 55,1987612104\rangle,\langle 56,63585120\rangle]$

Finally, the complete weight enumerators of the hull of $B 1$ :

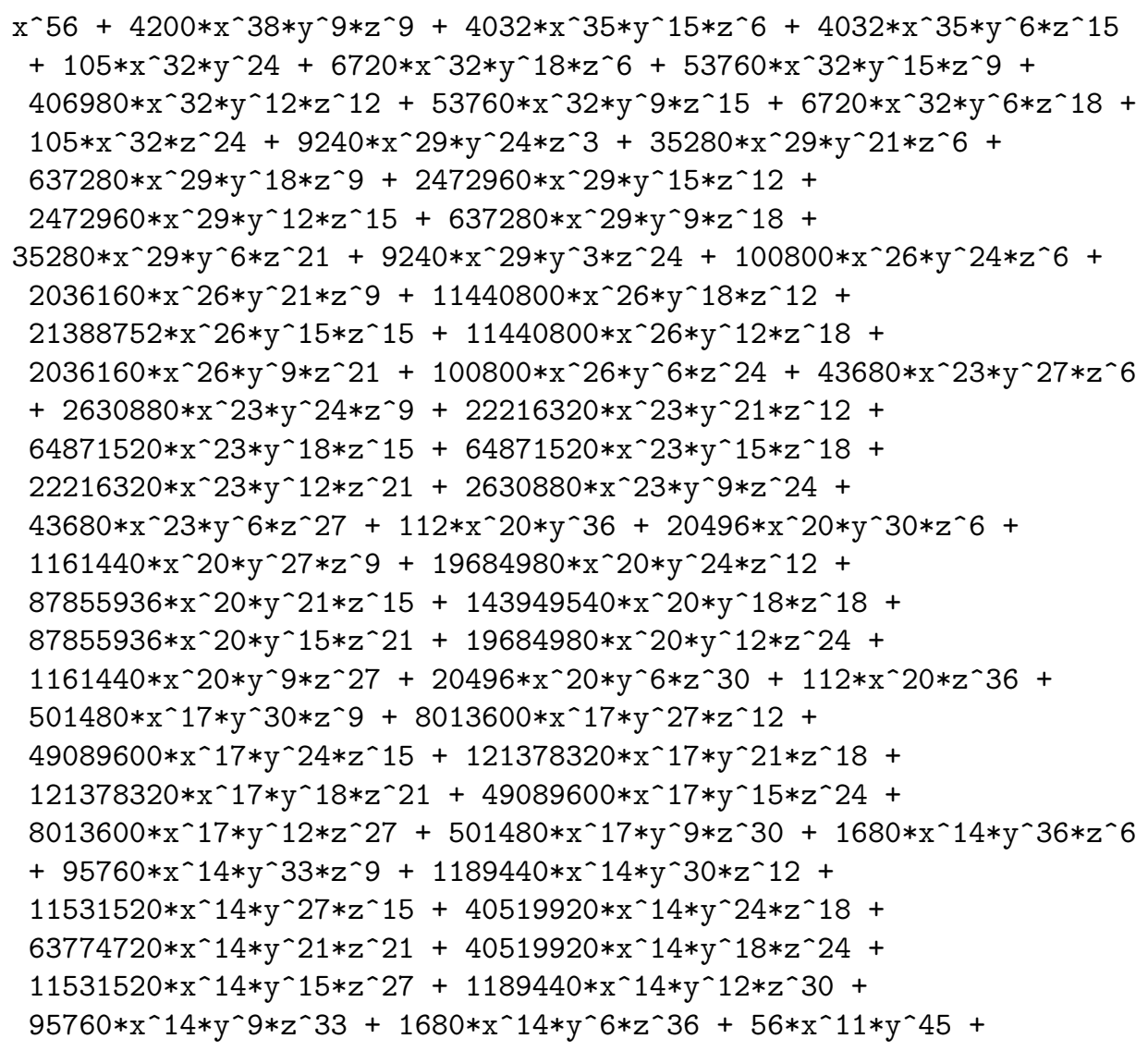




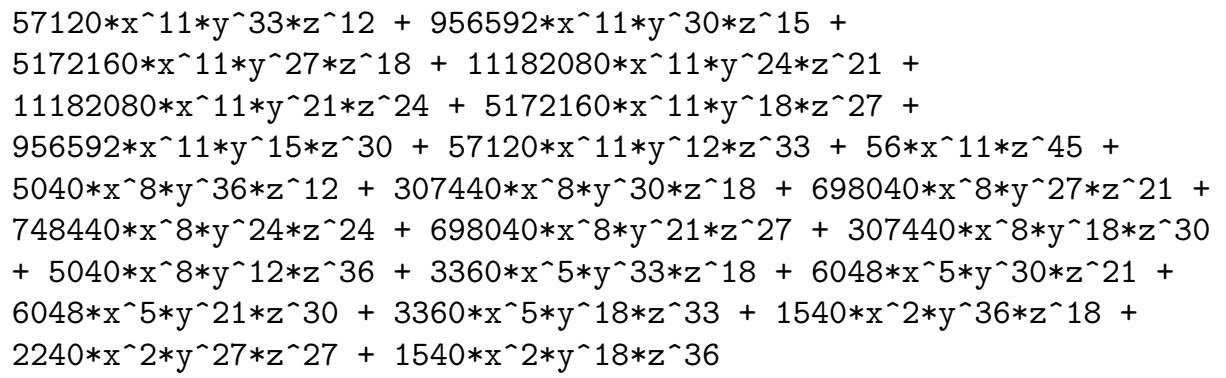

Total time: 11639.480 seconds

\subsection{The biplane $B 2$}

//weight distribution for the code (dim=22)

$[\langle 0,1\rangle,\langle 11,112\rangle,\langle 12,72\rangle,\langle 14,48\rangle,\langle 17,2592\rangle,\langle 18,10728\rangle$, $\langle 20,100312\rangle,\langle 21,166464\rangle,\langle 23,3113664\rangle,\langle 24,4392126\rangle,<26$, $53582976\rangle,\langle 27,58915312\rangle,\langle 29,476206960\rangle,\langle 30,429895296\rangle,\langle 32$, $2240452026\rangle,\langle 33,1628264160\rangle,\langle 35,5553150480\rangle,\langle 36,3239399268\rangle$, $\langle 38,6997381088\rangle,\langle 39,3230154000\rangle,\langle 41,4285622976\rangle,\langle 42$, $1530341856\rangle,\langle 44,1179228672\rangle,\langle 45,314249904\rangle,\langle 47,127445312\rangle$, $\langle 48,24103512\rangle,\langle 50,4361520\rangle,\langle 51,452160\rangle,\langle 53,57168\rangle,\langle 54$, $8344>,<56,500\rangle]$

//weight distribution for the code' orthogonal (dim=34)

$[\langle 0,1\rangle,\langle 8,702\rangle,\langle 10,6480\rangle,\langle 11,7680\rangle,\langle 12,157968\rangle,\langle 13$, $566784\rangle,\langle 14,3445776\rangle,\langle 15,17779104\rangle,\langle 16,89294346\rangle,\langle 17$, $411295392\rangle,\langle 18,1767595944\rangle,\langle 19,7083033120\rangle,\langle 20,26208252588\rangle$, $\langle 21,89936450208\rangle,\langle 22,286819820208\rangle,\langle 23,845530691760\rangle,\langle 24$, $2328746757426\rangle,\langle 25,5968767604032\rangle,\langle 26,14193918792144\rangle,<27$, $31584734994640\rangle,\langle 28,65516246755632\rangle,\langle 29,126168946108176\rangle,\langle 30$, 227417202907488>, <31, 381998632419648>, <32, 595235744351370>, $<33,866983570080768\rangle,\langle 34,1174582311869520\rangle,<35$, $1472572505770704\rangle,\langle 36,1720367145507780\rangle,\langle 37,1862413150806144\rangle$, $\langle 38,1857312479416488\rangle,\langle 39,1716790841485200\rangle,<40$, $1461269764802916\rangle,\langle 41,1137376311348816\rangle,\langle 42,813531480559632\rangle$, $\langle 43,530470354605984\rangle,\langle 44,312602259649968\rangle,<45$, 166949040057648>, <46, 79953846596496>, <47, 33929263511808>, <48, $12741046202988\rangle,\langle 49,4166256958560\rangle,\langle 50,1163453319720\rangle,<51$, $274157112384\rangle,\langle 52,52775804448\rangle,<53,7930805760\rangle,<54$, $878906344\rangle,<55,63096192\rangle,\langle 56,2301684\rangle]$ 
//weight distribution for the code's hull (dim=21)

$[<0,1\rangle,\langle 12,72\rangle,\langle 18,10728\rangle,\langle 21,166464\rangle,\langle 24,4392126\rangle$, $\langle 27,58915312\rangle,\langle 30,429895296\rangle,\langle 33,1628264160\rangle,\langle 36$, $3239399268\rangle,\langle 39,3230154000\rangle,\langle 42,1530341856\rangle,\langle 45$, $314249904\rangle,\langle 48,24103512\rangle,\langle 51,452160\rangle,\langle 54,8344\rangle]$

//weight distribution for the hull's orthogonal (dim=35)

$[\langle 0,1\rangle,\langle 7,192\rangle,\langle 8,702\rangle,\langle 10,7824\rangle,\langle 11,22416\rangle,\langle 12$, $323424\rangle,\langle 13,1397520\rangle,\langle 14,9178560\rangle,\langle 15,51540768\rangle,<16$, 262205238>, <17, 1230905088>, <18, 5309844744>, <19, $21267067008\rangle,\langle 20,78703622916\rangle,\langle 21,270269906400\rangle,\langle 22$, $858778650144\rangle,\langle 23,2538912673584\rangle,\langle 24,6991562585034\rangle,<25$, $17872963139856\rangle,\langle 26,42620848659792\rangle,\langle 27,94842691288528\rangle$, $<28,196193686785744\rangle,<29,378853947960864\rangle,<30$, $682875216407520\rangle,\langle 31,1143892791372096\rangle,<32$, $1787338009427868\rangle,\langle 33,2603325679478208\rangle,<34$, 3517325597732928>, <35, 4421775575483712>, <36, $5165827674280596>,<37,5577020371197456\rangle,<38$, $5577021278965416>,<39,5155076268144144>,<40$, $4375811579416272\rangle,\langle 41,3415270342093776\rangle,<42$, 2442830791079088>, <43, 1588499900043936>, <44, 938656309925376>, <45, 501302592634992〉, <46, 239425131238224>, $\langle 47,101884049612544\rangle,\langle 48,38259151526448\rangle$, $<49$, $12475490433264\rangle,\langle 50,3493028835576\rangle,\langle 51,822975137856\rangle,<52$, $158087937048\rangle,\langle 53,23847550128\rangle,\langle 54,2660458792\rangle,<55$, 193556832>, <56, 7263264> ]

Finally, the complete weight enumerator for the hull of $B 2$ :

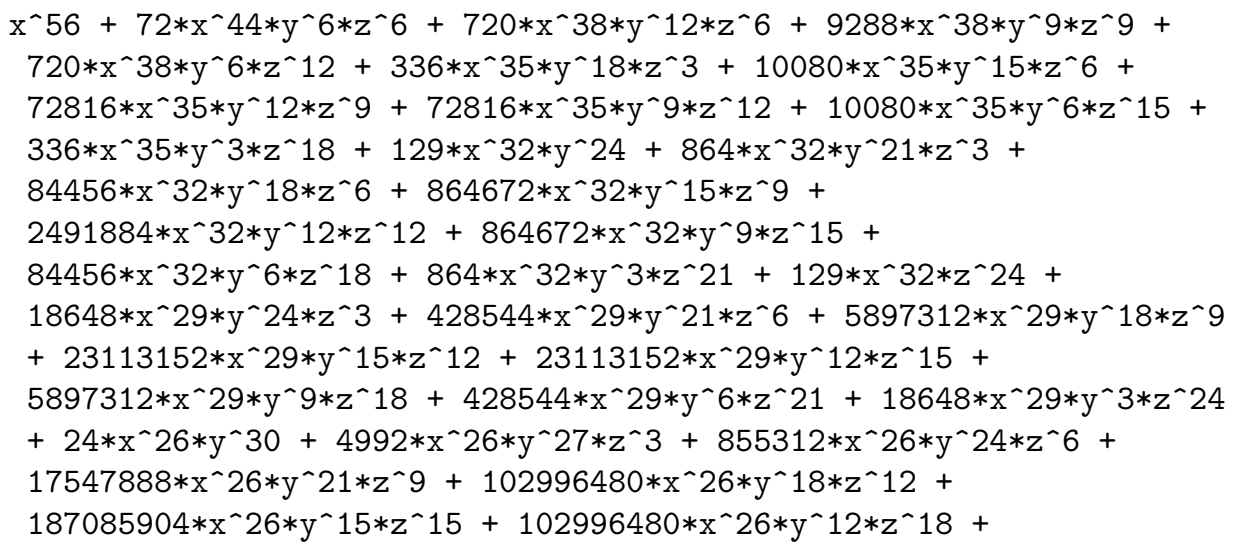




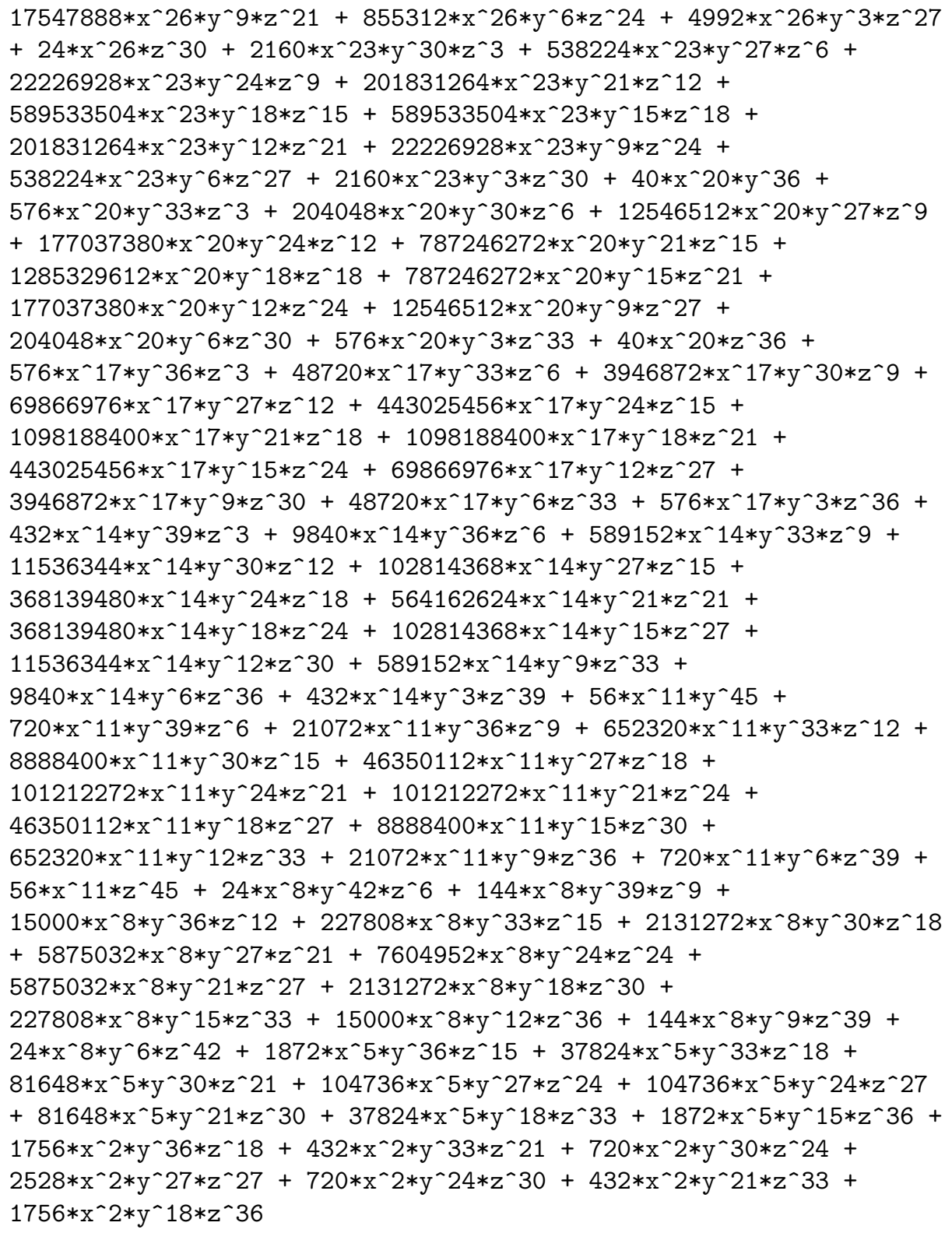

Total time: 110194.525 seconds

\subsection{The biplane $B 4$}

//weight distribution for the code (dim=24) 
$[\langle 0,1\rangle,\langle 11,112\rangle,\langle 12,304\rangle,\langle 14,480\rangle,\langle 15,1088\rangle,\langle 17,17856\rangle$, $\langle 18,52296\rangle,\langle 20,891272\rangle,\langle 21,1556672\rangle,\langle 23,28380000\rangle,\langle 24$, $39328678\rangle,\langle 26,481990800\rangle,\langle 27,533182480\rangle,\langle 29,4281444528\rangle,\langle 30$, $3858250128\rangle,\langle 32,20178805050\rangle,\langle 33,14672309952\rangle,<35$, $49958429040\rangle,\langle 36,29139602076\rangle,\langle 38,62987598416\rangle,<39$, $29076842096\rangle,\langle 41,38571782784\rangle,\langle 42,13772816896\rangle,\langle 44$,

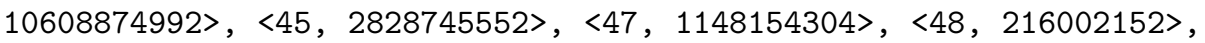
$\langle 50,39636096\rangle,\langle 51,4459520\rangle,\langle 53,351024\rangle,\langle 54,28936\rangle,\langle 56,900\rangle$ ]

// weight distribution for the hull (dim=23)

$[\langle 0,1\rangle,\langle 12,304\rangle,\langle 15,1088\rangle,\langle 18,52296\rangle,\langle 21,1556672\rangle,\langle 24$, $39328678\rangle,\langle 27,533182480\rangle,\langle 30,3858250128\rangle,\langle 33,14672309952\rangle$, $\langle 36,29139602076\rangle,\langle 39,29076842096\rangle,\langle 42,13772816896\rangle,\langle 45$, $2828745552\rangle,\langle 48,216002152\rangle,\langle 51,4459520\rangle,\langle 54,28936\rangle]$

//weight distribution of C's perp, (dim=32)

$[\langle 0,1\rangle,\langle 8,270\rangle,\langle 10,1360\rangle,\langle 11,1344\rangle,\langle 12,26152\rangle,\langle 13$, $67168\rangle,\langle 14,411280\rangle,\langle 15,1968544\rangle,\langle 16,10093138\rangle,\langle 17,45641440\rangle$, $\langle 18,194459752\rangle,\langle 19,794268704\rangle,\langle 20,2884485628\rangle,\langle 21$, 9987788384>, <22, 32225831888>, <23, 92910651920>, <24, $258790576154\rangle,<25,670444427744\rangle,\langle 26,1559689499984\rangle,<27$, $3509384014128\rangle,\langle 28,7359377720544\rangle,\langle 29,13864958016752\rangle,<30$, 25268482890672>, <31, 42909468111744>, <32, 65410371385458>, <33, $96331571833632\rangle,\langle 34,131939248029072\rangle,\langle 35,161820873965616\rangle,\langle 36$, 191152286658924>, <37, 209202793754144>, <38, 204100803000264>, <39, $190753730808560\rangle,\langle 40,164142412145924\rangle,\langle 41,124986106438064\rangle,\langle 42$, $90393085130000\rangle,\langle 43,59587222722848\rangle,\langle 44,34351896082208\rangle,\langle 45$, $18549594984272\rangle,\langle 46,8981057478064\rangle,\langle 47,3728553729152\rangle,\langle 48$, $1415729056876\rangle,\langle 49,468005702464\rangle,\langle 50,127833273736\rangle,\langle 51$, $30457665280\rangle,\langle 52,5926247728\rangle,\langle 53,871935616\rangle,\langle 54,98422616\rangle$, $\langle 55,7166400\rangle,\langle 56,280228\rangle]$

//weight distribution of B4's hull perp

$[\langle 0,1\rangle,\langle 7,96\rangle,\langle 8,270\rangle,\langle 10,2032\rangle,\langle 11,4880\rangle,\langle 12,59016\rangle$, $\langle 13,168720\rangle,\langle 14,1071216\rangle,\langle 15,5867040\rangle,\langle 16,29314446\rangle,\langle 17$,

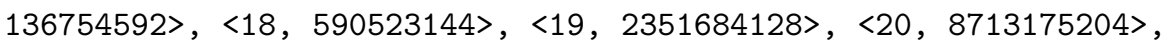
$\langle 21,30249442464\rangle,\langle 22,95079601056\rangle,\langle 23,281097688176\rangle,\langle 24$, $782577299202\rangle,\langle 25,1978566405072\rangle,\langle 26,4718166770208\rangle,\langle 27$, $10614911327920\rangle,\langle 28,21719707678336\rangle,\langle 29,41941003916672\rangle,<30$, 
$76430041491600\rangle,\langle 31,126633824377344\rangle,<32,197866621650852\rangle,<33$, 291371721912288>, <34, 389383450876992>, <35, 489509545064832>, <36, $578177957690028\rangle,\langle 37,617402329190192\rangle,\langle 38,617401776499576\rangle,<39$, $576970866889584\rangle,<40,484420422210960\rangle,<41,378086092928496\rangle,<42$, $273411057974064>,<43,175855054783584>,<44,103912802663232\rangle,<45$, $56106767113104\rangle,\langle 46,26505137407856\rangle,\langle 47,11279357161216\rangle,<48$, 4282247952048>, <49, 1381107601680>, <50, 386637072552>, <51, 92088444288>, <52, 17508342216>, <53, 2638080528>, <54, 299836024>, $<55,21722144\rangle,<56,864352\rangle]$

Finally, the complete weight enumerator for the hull of $B 4$ :

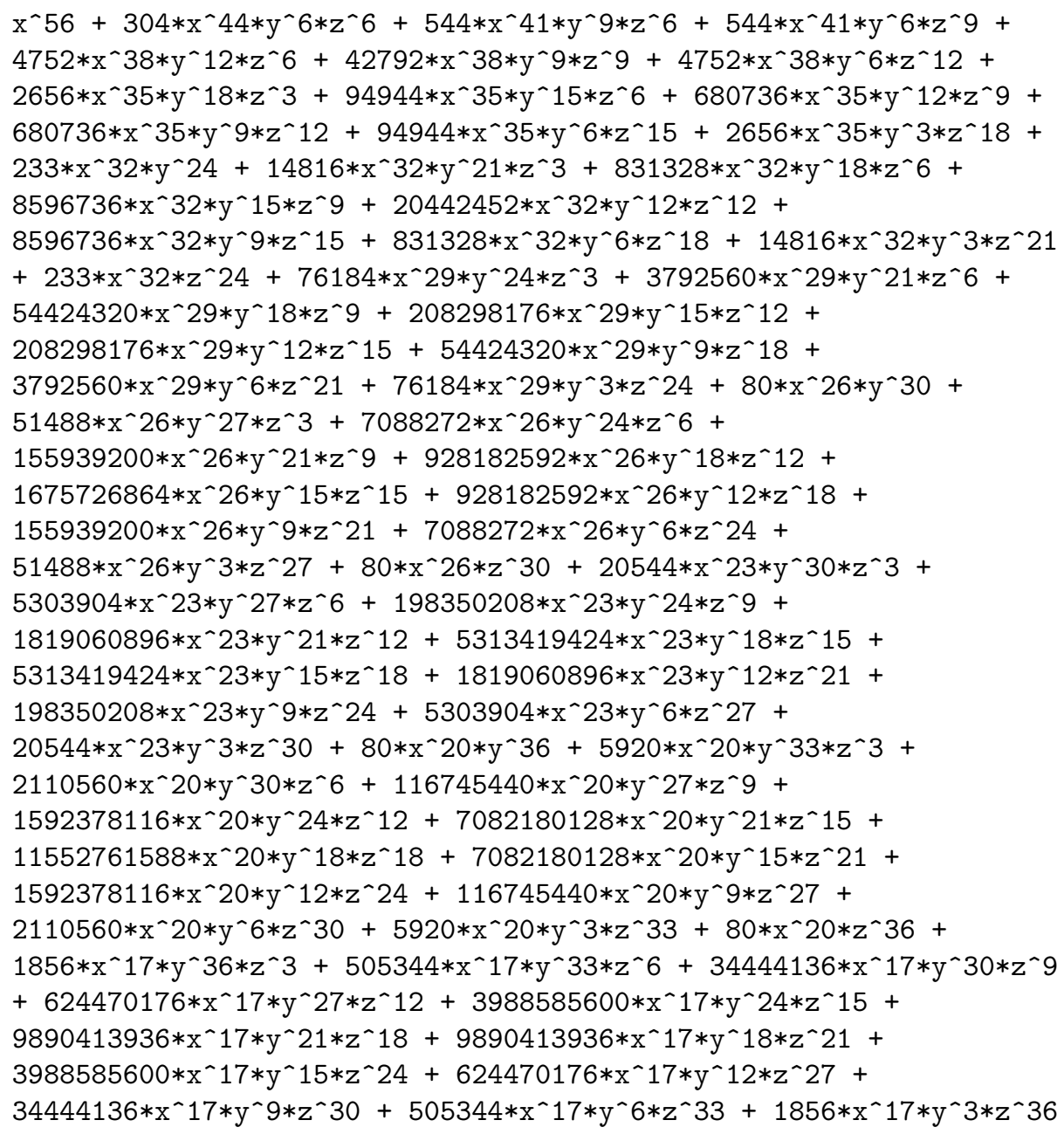




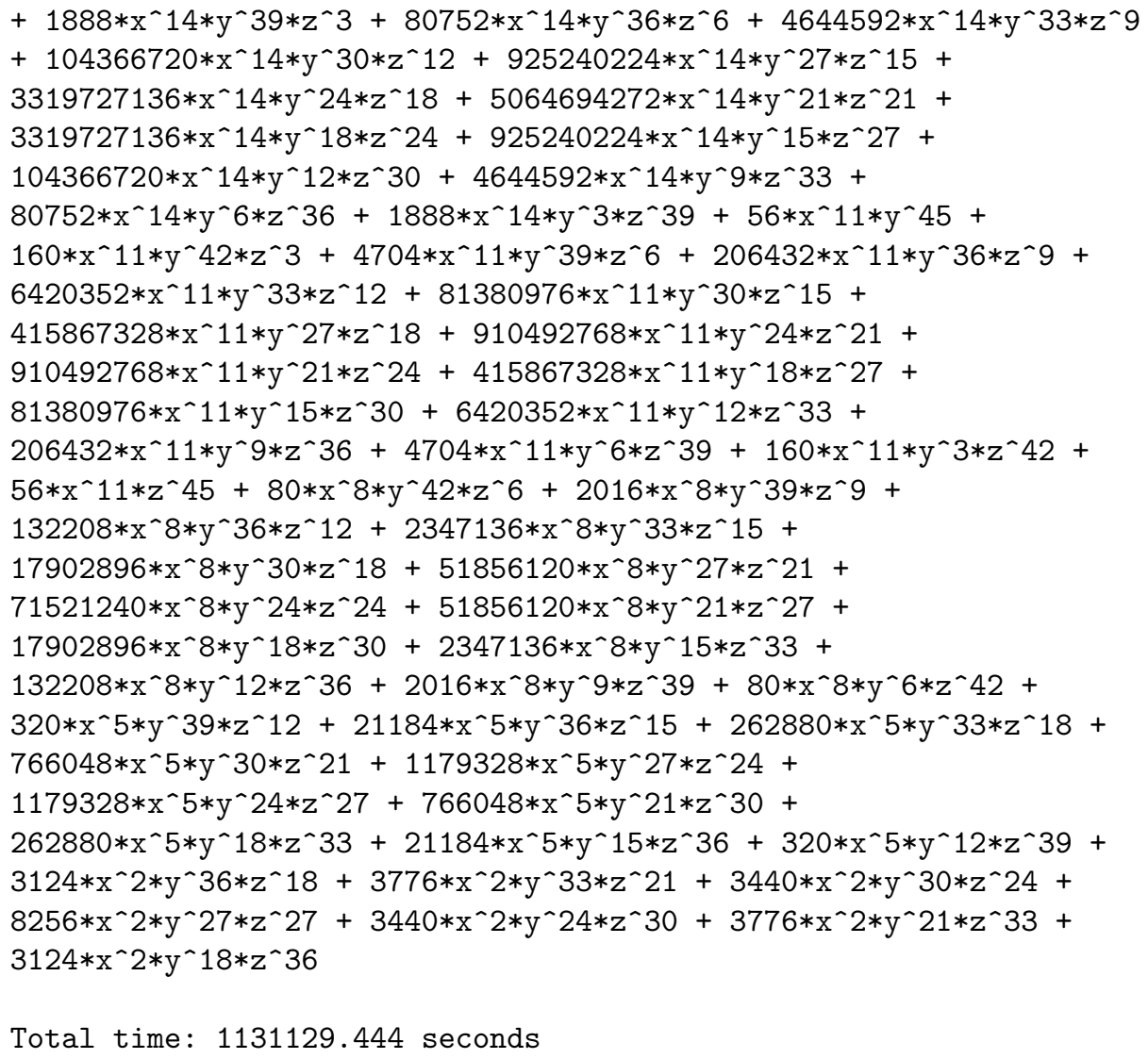

Total time: 1131129.444 seconds

\section{References}

[1] E. F. Assmus, Jr. and J. D. Key. Designs and their Codes. Cambridge University Press, 1992. Cambridge Tracts in Mathematics, Vol. 103 (Second printing with corrections, 1993).

[2] Bhaskar Bagchi. No extendable biplane of order 9. J. Combin. Theory, Ser. A, 49:1-12, 1988. Corrigendum in Vol. 57 (1991) p.162.

[3] Wieb Bosma and John Cannon. Handbook of Magma Functions. Department of Mathematics, University of Sydney, November 1994.

[4] Andries Brouwer. Private communication. 
[5] Andries E. Brouwer and Henny A. Wilbrink. Block designs. In F. Buekenhout, editor, Handbook of Incidence Geometry, pages 349382. Elsevier, 1995. Chapter 8.

[6] P. J. Cameron. Extending symmetric designs. J. Combin. Theory, Ser. A, 14:215-220, 1973.

[7] P. J. Cameron and J. H. van Lint. Designs, Graphs, Codes and their Links. Cambridge: Cambridge University Press, 1991. London Mathematical Society Student Texts 22 .

[8] R. H. F. Denniston. On biplanes with 56 points. Ars combin., 9:167179, 1980.

[9] M. Hall, Jr. and W.S. Connor. An embedding theorem for balanced incomplete block designs. Canad. J. Math., 6:35-41, 1954.

[10] M. Hall, Jr., R. Lane, and D. Wales. Designs derived from permutation groups. J. Combin. Theory, 8:12-22, 1970.

[11] D. R. Hughes and F. C. Piper. Design Theory. Cambridge: Cambridge University Press, 1985.

[12] Z. Janko and T. Van Trung. A new biplane of order 9 with a small automorphism group. J. Combin. Theory, Ser. A, 42:305-309, 1986.

[13] Elizabeth J. Morgan. Arcs in block designs. Ars Combin., 4:3-16, 1977.

[14] Chester J. Salwach and Joseph A. Mezzaroba. The four known biplanes with $k=11$. Internat. J. Math. \& Math. Sci., 2:251-260, 1979. 